\title{
Existence Theorems for a Fourth Order Boundary Value Problem
}

by

\author{
A. EL-HAFFAF \\ Presented by Czesław OLECH
}

Summary. This paper treats the question of the existence of solutions of a fourth order boundary value problem having the following form:

$$
\begin{aligned}
& x^{(4)}(t)+f\left(t, x(t), x^{\prime \prime}(t)\right)=0, \quad 0<t<1, \\
& x(0)=x^{\prime}(0)=0, \quad x^{\prime \prime}(1)=0, \quad x^{(3)}(1)=0 .
\end{aligned}
$$

Boundary value problems of very similar type are also considered. It is assumed that $f$ is a function from the space $C\left([0,1] \times \mathbb{R}^{2}, \mathbb{R}\right)$. The main tool used in the proof is the Leray-Schauder nonlinear alternative.

1. Introduction. Recently, fourth order boundary value problems have been considered in many papers. For example, Ma [20] studied the existence of positive solutions for the fourth order boundary value problem of the form

$$
\begin{aligned}
& x^{(4)}(t)=\lambda f\left(t, x(t), x^{\prime \prime}(t)\right), \quad 0<t<1, \\
& x(0)=x^{\prime}(0)=x^{\prime \prime}(1)=x^{(3)}(1)=0,
\end{aligned}
$$

under some sublinear semipositone conditions. The boundary conditions considered here are a special case of the $(p, n-p)$ right focal boundary conditions, in which $n=p=2$.

Extensive research has been done on focal boundary value problems. For some recent results, we refer the reader to the papers by Agarwal and O'Regan [1], Anderson [2], and Henderson and Kaufmann [16]. The fourth order boundary value problems have been studied by many authors, for example Yang [22], Kosmatov [18], and Graef, Qian and Yang [10]. Most

2000 Mathematics Subject Classification: 34B15, 34B14, 34B10.

Key words and phrases: Green's function, fixed point theorem, Leray-Schauder nonlinear alternative, lower and upper solutions. 
of them studied the existence of positive solutions for the equation

$$
\begin{aligned}
& x^{(4)}(t)=g(t) f(x), \quad 0<t<1, \\
& x(0)=x^{\prime}(0)=x^{\prime \prime}(1)=x^{\prime \prime}(1)=0, \\
& \text { or } \quad x(0)=x(1)=x^{\prime}(0)=x^{\prime}(1)=0 .
\end{aligned}
$$

Recent contributions on the existence of multiple positive solutions include the papers of Henderson, Graef and Yang [9] and Henderson and Ntouyas [17].

The method of lower and upper solutions has been extensively developed for lower order equations with linear and nonlinear boundary conditions. But there are only a few applications to higher order ordinary differential equations. In this paper, the lower and upper solutions method is employed to obtain the existence results for some boundary value problems with monotonic or quasi-monotonic nonlinear term $f$ which does not depend on any lower order derivatives.

2. Preliminaries. This paper is organized as follows. In Section 2, we present two lemmas that will be used to prove the main results. In Section 3, we obtain some results on existence of nontrivial solution of the BVP (1.1) without imposing any monotonicity and nonnegativity conditions on $f$. Finally, in Section 4, we study the existence of solutions for two classes of nonlinear fourth order boundary value problems (1.1) and also very similar boundary value problems. The method used here is based on the Schauder fixed-point theorem and the lower and upper solutions method. The construction of lower or upper solutions is also presented.

We consider the BVP (1.1) under the assumption that $f \in C([0,1] \times$ $\left.\mathbb{R}^{2}, \mathbb{R}\right)$. Let $E=C^{4}[0,1]$ with $\|x\|=\max \left\{|x|_{\infty},\left|x^{\prime \prime}\right|_{\infty}\right\}$ where $|x|_{\infty}=$ $\max _{t \in[0,1]}|x(t)|$ for any $x \in E$.

Lemma 2.1 ([22]). Let $y \in C[0,1]$. Then the four-point $B V P$

$$
\begin{aligned}
& x^{(4)}+y(t)=0, \quad 0<t<1, \\
& x(0)=x^{\prime}(0)=x^{\prime \prime}(1)=x^{(3)}(1)=0,
\end{aligned}
$$

is equivalent to the integral equation

$$
x(t)=\int_{0}^{1} G(t, s) y(s) d s
$$

where $G:[0,1] \times[0,1] \rightarrow[0, \infty)$ denotes the Green function given by

$$
G(t, s)= \begin{cases}\frac{1}{6} t^{2}(3 s-t), & 0 \leq t \leq s \leq 1, \\ \frac{1}{6} s^{2}(3 t-s), & 0 \leq s \leq t \leq 1,\end{cases}
$$


Define the integral operator $T: E \rightarrow E$ by

$$
T x(t)=\frac{1}{6} \int_{0}^{t} s^{2}(3 t-s) y(s) d s+\frac{1}{6} \int_{t}^{1} t^{2}(3 s-t) y(s) d s .
$$

By Lemma 2.1, the BVP (1.1) has a solution if and only if the operator $T$ has a fixed point in $E$. So we only need to seek a fixed point of $T$ in $E$. By the Ascoli-Arzelà theorem, we can prove that $T$ is a completely continuous operator. The key tool in our approach is the following Leray-Schauder nonlinear alternative:

Lemma 2.2 ([6]). Let $E$ be a Banach space and $\Omega$ be a bounded open subset of $E$ with $0 \in \Omega$. Let $T: \bar{\Omega} \rightarrow E$ be a completely continuous operator. Then, either

(i) there exist $x \in \partial \Omega$ and $\lambda>1$ such that $T(x)=\lambda x$, or

(ii) there exists a fixed point $x^{*} \in \bar{\Omega}$.

3. Existence of nontrivial solutions. In this section, we prove the existence of a nontrivial solution for the BVP (1.1). Suppose that $f \in$ $C\left([0,1] \times \mathbb{R}^{2}, \mathbb{R}\right)$.

TheOREM 3.1. Suppose that $f(t, 0,0) \not \equiv 0$ and there exist nonnegative functions $k, h, l \in L^{1}[0,1]$ such that

$$
|f(t, x, y)| \leq k(t)|x|+h(t)|y|+l(t), \quad \text { a.e. }(t, x, y) \in[0,1] \times \mathbb{R}^{2},
$$

and

$$
\int_{0}^{1}\left(\frac{1}{2} s^{2}+\frac{1}{3} s^{3}\right)(k(s)+h(s)) d s<1 .
$$

Then the BVP (1.1) has at least one nontrivial solution $x^{*} \in C^{4}[0,1]$.

Proof. Let

$$
\begin{aligned}
M & =\int_{0}^{1}\left(\frac{1}{2} s^{2}+\frac{1}{3} s^{3}\right)(k(s)+h(s)) d s, \\
N & =\int_{0}^{1}\left(\frac{1}{2} s^{2}+\frac{1}{3} s^{3}\right) l(s) d s .
\end{aligned}
$$

Then $M<1$. Since $f(t, 0,0) \not \equiv 0$, there exists an interval $[a, b] \subset[0,1]$ such that $\min _{a \leq t \leq b}|f(t, 0,0)|>0$. On the other hand, from $l(t) \geq|f(t, 0,0)|$, a.e. $t \in[0,1]$, we know that $N>0$.

Let $A=N(1-M)^{-1}$ and $\Omega=\{x \in E:\|x\|<A\}$. Suppose $x \in \partial \Omega$ and $\lambda>1$ are such that $T x=\lambda x$. Then 


$$
\begin{aligned}
\lambda A & =\lambda\|x\|=\|T x\|=\max _{0 \leq t \leq 1}|(T x)(t)| \\
& \leq \frac{1}{6} \int_{0}^{t} s^{2}(3 t-s)\left|f\left(s, x(s), x^{\prime \prime}(s)\right)\right| d s+\frac{1}{6} \int_{t}^{1} t^{2}(3 s-t)\left|f\left(s, x(s), x^{\prime \prime}(s)\right)\right| d s \\
& \leq \frac{1}{6} \int_{0}^{1} s^{2}(3-s)\left|f\left(s, x(s), x^{\prime \prime}(s)\right)\right| d s+\frac{1}{6} \int_{0}^{1} s^{2}(3 s)\left|f\left(s, x(s), x^{\prime \prime}(s)\right)\right| d s \\
& \leq \frac{1}{6} \int_{0}^{1}\left(s^{2}(3-s)+3 s^{3}\right)\left|f\left(s, x(s), x^{\prime \prime}(s)\right)\right| d s \\
& \leq \int_{0}^{1}\left(\frac{1}{2} s^{2}+\frac{1}{3} s^{3}\right)\left(k(s)|x(s)|+h(s)\left|x^{\prime \prime}(s)\right|+l(s)\right) d s \\
& \leq \int_{0}^{1}\left(\frac{1}{2} s^{2}+\frac{1}{3} s^{3}\right)\left(k(s) \max _{0 \leq s \leq 1}|x(s)|+h(s) \underset{0 \leq s \leq 1}{\max }\left|x^{\prime \prime}(s)\right|+l(s)\right) d s \\
& \leq \int_{0}^{1}\left(\frac{1}{2} s^{2}+\frac{1}{3} s^{3}\right)\left(k(s)|x|_{\infty}+h(s)\left|x^{\prime \prime}\right| \infty+l(s)\right) d s \\
& \leq \int_{0}^{1}\left(\frac{1}{2} s^{2}+\frac{1}{3} s^{3}\right)(k(s)\|x\|+h(s)\|x\|+l(s)) d s \\
& \left.\quad s^{2}+\frac{1}{3} s^{3}\right)(k(s)+h(s))\|x\| d s+\int_{0}^{1}\left(\frac{1}{2} s^{2}+\frac{1}{3} s^{3}\right) l(s) d s \\
& \leq x .
\end{aligned}
$$

Therefore,

$$
\lambda \leq M+\frac{N}{A}=M+\frac{N}{N(1-M)^{-1}}=1+(1-M)=1 .
$$

This contradicts $\lambda>1$. By Lemma 2.2, $T$ has a fixed point $x^{*} \in \bar{\Omega}$. In view of $f(t, 0,0) \not \equiv 0$, the $\operatorname{BVP}(1.1)$ has a nontrivial solution $x^{*} \in E$. This completes the proof.

THEOREM 3.2. Suppose that $f(t, 0,0) \not \equiv 0$, and there exist nonnegative functions $k, h, l \in L^{1}[0,1]$ such that

$$
|f(t, x, y)| \leq k(t)|x|+h(t)|y|+l(t), \quad \text { a.e. }(t, x, y) \in[0,1] \times \mathbb{R}^{2} .
$$

Assume that one of the following conditions holds:

(1) There exists a constant $\alpha>-3$ such that

$$
k(s)+h(s) \leq \frac{6(3+\alpha)(4+\alpha)}{18+5 \alpha} s^{\alpha}, \quad \text { a.e. } s \in[0,1],
$$




$$
\text { meas }\left\{s \in[0,1]: k(s)+h(s)<\frac{6(3+\alpha)(4+\alpha)}{18+5 \alpha} s^{\alpha}\right\}>0 .
$$

(2) There exists a constant $\alpha>-1$ such that

$$
\begin{aligned}
& k(s)+h(s) \leq \frac{6+\alpha}{(1+\alpha)(2+\alpha)(3+\alpha)(4+\alpha)}(1-s)^{\alpha}, \quad \text { a.e. } s \in[0,1], \\
& \operatorname{meas}\left\{s \in[0,1]: k(s)+h(s)<\frac{6+\alpha}{(1+\alpha)(2+\alpha)(3+\alpha)(4+\alpha)}(1-s)^{\alpha}\right\}>0 .
\end{aligned}
$$

(3) There exists a constant $p>1$ such that

$$
\int_{0}^{1}(k(s)+h(s))^{p} d s<\left[\frac{1}{\frac{1}{2}\left(\frac{1}{2 q+1}\right)^{1 / q}+\frac{1}{3}\left(\frac{1}{3 q+1}\right)^{1 / q}}\right]^{p} \quad\left(\frac{1}{p}+\frac{1}{q}=1\right) .
$$

Then the BVP (1.1) has at least one nontrivial solution $x^{*} \in E$.

Proof. Let $M$ be as in the proof of Theorem 3.1. In view of Theorem 3.1, we only need to prove that $M<1$.

(1) In this case, we have

$$
\begin{aligned}
M & <\frac{6(3+\alpha)(4+\alpha)}{18+5 \alpha} \int_{0}^{1}\left(\frac{1}{2} s^{2}+\frac{1}{3} s^{3}\right) s^{\alpha} d s \\
& =\frac{6(3+\alpha)(4+\alpha)}{18+5 \alpha} \int_{0}^{1}\left(\frac{1}{2} s^{\alpha+2}+\frac{1}{3} s^{3+\alpha}\right) d s \\
& <\frac{6(3+\alpha)(4+\alpha)}{18+5 \alpha}\left[\frac{1}{2(3+\alpha)}+\frac{1}{3(4+\alpha)}\right]=1 .
\end{aligned}
$$

(2) In this case, we have

$$
\begin{aligned}
M \leq & \frac{6+\alpha}{(1+\alpha)(2+\alpha)(3+\alpha)(4+\alpha)} \int_{0}^{1}\left(\frac{1}{2} s^{2}+\frac{1}{3} s^{3}\right)(1-s)^{\alpha} d s \\
< & \frac{6+\alpha}{(1+\alpha)(2+\alpha)(3+\alpha)(4+\alpha)} \\
& \times\left(\int_{0}^{1}\left(\frac{1}{2} s^{2}\right)(1-s)^{\alpha} d s+\int_{1}^{1}\left(\frac{1}{3} s^{3}\right)(1-s)^{\alpha} d s\right) .
\end{aligned}
$$

A simple computation (integration by parts) yields

$$
\begin{aligned}
M< & \frac{6+\alpha}{(1+\alpha)(2+\alpha)(3+\alpha)(4+\alpha)} \\
& \times\left(\frac{1}{(1+\alpha)(2+\alpha)(3+\alpha)}+\frac{2}{(1+\alpha)(2+\alpha)(3+\alpha)(4+\alpha)}\right)=1 .
\end{aligned}
$$


(3) Using the Hölder inequality, we have

$$
\begin{aligned}
M & \leq\left[\int_{0}^{1}(k(s)+h(s))^{p} d s\right]^{1 / p}\left\{\frac{1}{2}\left[\int_{0}^{1}\left(s^{2}\right)^{q} d s\right]^{1 / q}+\frac{1}{3}\left[\int_{0}^{1}\left(s^{3}\right)^{q} d s\right]^{1 / q}\right\} \\
& \leq\left[\int_{0}^{1}(k(s)+h(s))^{p} d s\right]^{1 / p}\left\{\frac{1}{2}\left(\frac{1}{2 q+1}\right)^{1 / q}+\frac{1}{3}\left(\frac{1}{3 q+1}\right)^{1 / q}\right\} \\
& \leq\left[\frac{1}{\frac{1}{2}\left(\frac{1}{2 q+1}\right)^{1 / q}+\frac{1}{3}\left(\frac{1}{3 q+1}\right)^{1 / q}}\right]\left\{\frac{1}{2}\left(\frac{1}{2 q+1}\right)^{1 / q}+\frac{1}{3}\left(\frac{1}{3 q+1}\right)^{1 / q}\right\} \\
& =1 .
\end{aligned}
$$

This completes the proof.

4. Examples. In order to illustrate the above results, we consider some examples.

Example 4.1. Consider the following problem:

$$
\begin{aligned}
& x^{(4)}+\frac{2 \sqrt{t} x^{3}}{5+x^{4}} e^{-\left|\cos \left(x^{2}-t\right)\right|}+\frac{t^{2}\left(x^{\prime \prime}\right)^{2} e^{-\left(x^{\prime \prime}\right)^{2}}}{t^{2}+\left(x^{\prime \prime}\right)^{2}}+2 e^{t}-3 \sin t=0, \\
& x(0)=x^{\prime}(0)=0, \quad x^{\prime \prime}(1)=x^{(3)}(1) .
\end{aligned}
$$

Set

$$
\begin{aligned}
f(t, x, y) & =\frac{2 \sqrt{t} x^{3}}{5+x^{4}} e^{-\left|\cos \left(x^{2}-t\right)\right|}+\frac{t^{2} y^{2} e^{-y^{2}}}{t^{2}+y^{2}}+2 e^{t}-3 \sin t, \\
k(t) & =\sqrt{\frac{t}{5}}, \quad h(t)=\frac{t}{2}, \quad l(t)=2 e^{t}+3 \sin t .
\end{aligned}
$$

It is easy to prove that $k, h, l \in L^{1}[0,1]$ are nonnegative functions and

$$
|f(t, x, y)| \leq k(t)|x|+h(t)|y|+l(t), \quad(t, x, y) \in[0,1] \times \mathbb{R}^{2} .
$$

Moreover, we have

$$
M=\int_{0}^{1}\left(\frac{1}{2} s^{2}+\frac{1}{3} s^{3}\right)(k(s)+h(s)) d s=\frac{1}{4 \sqrt{5}}+\frac{23}{240}<1 .
$$

Hence, by Theorem 3.1, problem (4.1) has at least one nontrivial solution.

EXAMPLE 4.2. Let us consider the problem

$$
\begin{aligned}
& x^{(4)}+\frac{x^{2}}{3\left(1+x^{2}\right) \sqrt{t}} e^{-x^{2}}+\frac{\left(x^{\prime \prime}\right)^{2} e^{-t}}{5\left(1+\left(x^{\prime \prime}\right)^{2}\right)\left(1+3 e^{x^{\prime \prime}}\right) \sqrt{t}} \\
& +\frac{1}{3 \sqrt{t}} x^{\prime \prime}-e^{t}+\sqrt{\sin t}=0, \\
& x(0)=x^{\prime}(0)=0, \quad x^{\prime \prime}(1)=x^{(3)}(1)=0 .
\end{aligned}
$$


Set

$$
\begin{gathered}
f(t, x, y)=\frac{x^{2}}{3\left(1+x^{2}\right) \sqrt{t}} e^{-x^{2}}+\frac{y^{2} e^{-t}}{5\left(1+y^{2}\right)\left(1+3 e^{y}\right) \sqrt{t}} \\
\quad+\frac{1}{3 \sqrt{t}} y-e^{t}+\sqrt{\sin t}, \\
k(t)=\frac{1}{6 \sqrt{t}}, \quad h(t)=\frac{1}{15 \sqrt{t}}+\frac{1}{3 \sqrt{t}}, \quad l(t)=2 e^{t}+3 \sin t .
\end{gathered}
$$

It is easy to prove that $k, h, l \in L^{1}[0,1]$ are nonnegative functions and

$$
|f(t, x, y)| \leq k(t)|x|+h(t)|y|+l(t), \quad \text { a.e. }(t, x, y) \in[0,1] \times \mathbb{R}^{2} .
$$

Let $\alpha=-1 / 2$. Then

$$
\frac{6(3+\alpha)(4+\alpha)}{18+5 \alpha}=\frac{105}{31}
$$

Therefore,

$$
\begin{gathered}
k(s)+h(s)=\frac{1}{6 \sqrt{s}}+\frac{1}{15 \sqrt{s}}+\frac{1}{3 \sqrt{s}}=\frac{1}{6 \sqrt{s}}+\frac{2}{5 \sqrt{s}} \leq \frac{3}{5} s^{-1 / 2}<\frac{105}{31} s^{-1 / 32}, \\
\operatorname{meas}\left\{s \in[0,1]: k(s)+h(s)<\frac{6(3+\alpha)(4+\alpha)}{18+5 \alpha} s^{\alpha}\right\}>0 .
\end{gathered}
$$

Hence, by Theorem 3.2(1), problem (4.2) has at least one nontrivial solution.

ExAmple 4.3. Consider the problem

$$
\begin{aligned}
& x^{(4)}+\frac{x^{2} e^{-t^{3} / 3}}{2\left(1+x^{2}\right)\left(1+7 e^{x}\right) \sqrt[4]{1-t}} x+\frac{1}{7 \sqrt[4]{1-t}} \\
& +\frac{\left(x^{\prime \prime}\right)^{4}}{\left(3+\left(x^{\prime \prime}\right)^{5}\right) \sqrt[4]{1-t}} e^{-\left|\sin \left(x^{\prime \prime}-t\right)\right|}-3 e^{-t}+3 \sin ^{2} t=0, \\
& x(0)=x^{\prime}(0)=0, \quad x^{\prime \prime}(1)=x^{(3)}(1)=0 .
\end{aligned}
$$

Set

$$
\begin{gathered}
f(t, x, y)=\frac{x^{2} e^{-t^{3} / 3}}{2\left(1+x^{2}\right)\left(1+7 e^{x}\right) \sqrt[4]{1-t}} x+\frac{1}{7 \sqrt[4]{1-t}} \\
+\frac{l(y)^{4}}{\left(3+(y)^{5}\right) \sqrt[4]{1-t}} e^{-|\sin (y-t)|}-3 e^{-t}+3 \sin ^{2} t, \\
k(t)=\frac{1}{14 \sqrt[4]{1-t}}+\frac{1}{7 \sqrt[4]{1-t}}, \quad h(t)=\frac{1}{3 \sqrt[4]{1-t}}, \quad l(t)=3 e^{-t}+3 \sin ^{2} t .
\end{gathered}
$$

It is easy to prove that $k, h, l \in L^{1}[0,1]$ are nonnegative functions and

$$
|f(t, x, y)| \leq k(t)|x|+h(t)|y|+l(t), \quad \text { a.e. }(t, x, y) \in[0,1] \times \mathbb{R}^{2} .
$$


Let $\alpha=-1 / 4$. Then

$$
\frac{6+\alpha}{(1+\alpha)(2+\alpha)(3+\alpha)(4+\alpha)}=\frac{1600}{3465} .
$$

Therefore,

$$
\begin{aligned}
k(s)+h(s) & =\frac{1}{14 \sqrt[4]{1-s}}+\frac{1}{7 \sqrt[4]{1-s}}+\frac{1}{3 \sqrt[4]{1-s}} \\
& =\frac{10}{42 \sqrt[4]{1-s}}<\frac{1600}{3465}(1-s)^{-1 / 4} .
\end{aligned}
$$

Hence, by Theorem 3.2(2), problem (4.3) has at least one nontrivial solution.

EXAmple 4.4. Consider the problem

$$
\begin{aligned}
& \begin{array}{l}
x^{(4)}+(\sqrt{t}-t)|x| \cos x-t x+\frac{3 \sqrt{t}\left(x^{\prime \prime}\right)^{2} e^{-\left|\sin \left(\left(x^{\prime \prime}\right)^{2}-t\right)\right|}}{4+\left(x^{\prime \prime}\right)^{3}} \\
+\sin \left(e^{t}\right)+3 \cos ^{2} t=0, \\
x(0)=x^{\prime}(0)=0, \quad x^{\prime \prime}(1)=x^{(3)}(1)=0 .
\end{array}
\end{aligned}
$$

Set

$$
\begin{array}{r}
f(t, x, y)=x^{(4)}+(\sqrt{t}-t)|x| \cos x-t x \\
\quad+\frac{3 \sqrt{t} y^{2} e^{-\left|\sin \left(y^{2}-t\right)\right|}}{\left(4+y^{3}\right)}+\sin \left(e^{t}\right)+3 \cos ^{2} t \\
k(t)=\sqrt{t}, \quad h(t)=\sqrt{t}, \quad l(t)=\sin \left(e^{t}\right)+3 \cos ^{2} t .
\end{array}
$$

It is easy to prove that $k, h, l \in L^{1}[0,1]$ are nonnegative functions and

$$
|f(t, x, y)| \leq k(t)|x|+h(t)|y|+l(t), \quad \text { a.e. }(t, x, y) \in[0,1] \times \mathbb{R}^{2} .
$$

Let $p=q=2$. Then

$$
\int_{0}^{1}(k(s)+h(s))^{p} d s=\int_{0}^{1}(2 \sqrt{t})^{2}=2 .
$$

Moreover, we have

$$
\begin{aligned}
2 & <\left[\frac{1}{\frac{1}{2}\left(\frac{1}{2 q+1}\right)^{1 / q}+\frac{1}{3}\left(\frac{1}{3 q+1}\right)^{1 / q}}\right]^{p}=\left[\frac{1}{\frac{1}{2}\left[\frac{1}{5}\right]^{1 / 2}+\frac{1}{3}\left[\frac{1}{7}\right]^{1 / 2}}\right]^{2} \\
& =\frac{1260}{(3 \sqrt{7}+2 \sqrt{5})^{2}} .
\end{aligned}
$$

Hence, by Theorem 3.2(3), problem (4.4) has at least one nontrivial solution.

5. Upper and lower solutions method. The purpose of this section is to study the existence of solution for two nonlinear fourth order boundary 
value problems

$$
\begin{aligned}
& x^{(4)}(t)+f\left(t, x(t), x^{\prime \prime}(t)\right)=0, \quad 0<t<1, \\
& x(0)=x^{\prime}(0)=x^{\prime \prime}(1)=x^{(3)}(0)=0,
\end{aligned}
$$

and

$$
\begin{aligned}
& x^{(4)}(t)+f\left(t, x(t), x^{\prime \prime}(t), x^{(3)}(t)\right)=0, \quad 0<t<1, \\
& x(0)=x^{\prime}(0)=x^{\prime \prime}(1)=x^{(3)}(0)=0 .
\end{aligned}
$$

We consider (5.1) under the assumption that $f:[0,1] \times \mathbb{R}^{2} \rightarrow \mathbb{R}$ is continuous. We give some lemmas which indicate some restrictions on the nonlinear term and let us construct lower and upper solutions.

Definition 5.1. We call $\alpha, \beta \in C^{3}[0,1] \cap C^{4}(0,1)$ a lower and an upper solution of problem (5.1), respectively, if

$$
\begin{aligned}
& \alpha^{(4)}(t)+f\left(t, \alpha(t), \alpha^{\prime \prime}(t)\right) \geq 0, \quad 0<t<1, \\
& \alpha(0)=\alpha^{\prime}(0)=\alpha^{\prime \prime}(1)=0, \quad \alpha^{(3)}(0) \geq 0, \\
& \beta^{(4)}(t)+f\left(t, x(t), x^{\prime \prime}(t)\right) \leq 0, \quad 0<t<1, \\
& \beta(0)=\beta^{\prime}(0)=\beta^{\prime \prime}(1)=0, \quad \beta^{(3)}(0) \leq 0 .
\end{aligned}
$$

Denote by $G(t, s)$ the Green's function of the following BVP:

$$
\begin{array}{ll}
-x^{(3)}(t)=0, & 0<t<1, \\
x(0)=x^{\prime}(0), & x^{\prime \prime}(1)=0 .
\end{array}
$$

Then

$$
G(t, s)= \begin{cases}\frac{1}{2} t^{2}, & 0 \leq t \leq s \leq 1 \\ s t-\frac{1}{2} s^{2}, & 0 \leq s \leq t \leq 1\end{cases}
$$

Lemma 5.2. For $G(t, s)$ defined by (5.3), the following holds:

(1) $G(t, s) / G(s, s) \leq 1$ for $t, s \in(0,1)$,

(2) $G(t, s) / G(s, s)>0$ for $t, s \in(0,1)$.

The proof of Lemma 5.2 is very straightforward and therefore omitted.

Let $\ell=\int_{0}^{1} G(s, s) s d s$. It is easy to see that $\ell=1 / 8$.

LEMMA 5.3. If there exists a constant $A \geq 0$ such that

$$
f(t, p, q) \leq A \quad \text { for } 0 \leq t \leq 1,0 \leq p \leq \ell A, 0 \leq q \leq A / 2,
$$

then problem (5.1) has an upper solution.

Proof. Set $u(t)=-x^{(3)}(t)$. Then problem (5.1) is equivalent to

$$
\begin{aligned}
& u^{\prime}(t)=f(t,(T u)(t),(K u)(t)), \quad 0<t<1, \\
& u(0)=0,
\end{aligned}
$$


where $(T u)(t)=\int_{0}^{1} G(t, s) u(s) d s, x^{\prime \prime}(t)=\int_{t}^{1} u(s) d s=(K u)(t)$ and $G(t, s)$ is the Green's function defined by (5.4). It is clear that the restriction on $f$ guarantees that $\psi(t)=A t$ satisfies the first order equation

$$
\begin{aligned}
& \psi^{\prime}(t)-f(t,(T \psi)(t),(K \psi)(t)) \geq 0, \quad 0<t<1, \\
& \psi(0) \geq 0 .
\end{aligned}
$$

This shows that $\beta(t)=(T \psi)(t)$ is an upper solution of problem (5.1).

LEMMA 5.4. If there exists a constant $B \leq 0$ such that

$$
f(t, p, q) \geq B \quad \text { for } 0 \leq t \leq 1, \ell B \leq p \leq 0,0 \leq q \leq-B / 2,
$$

then problem (5.1) has a lower solution.

Proof. Set $\phi(t)=B t$. Then it is clear that the restriction on $f$ guarantees that

$$
\begin{aligned}
& \phi^{\prime}(t)-f(t,(T \phi)(t),(K \phi)(t)) \leq 0, \quad 0<t<1, \\
& \phi(0) \leq 0 .
\end{aligned}
$$

This shows that $\alpha(t)=(T \phi)(t)$ is a lower solution of problem (5.1).

REMARK 5.5. Explicitly, we can write the upper solution $\beta(t)$ and lower solution $\alpha(t)$ of problem (5.1) as

$$
\beta(t)=-\frac{A}{24} t^{4}+\frac{A}{4} t^{2}, \quad \alpha(t)=-\frac{B}{24} t^{4}+\frac{B}{4} t^{2} .
$$

TheOrem 5.6. Suppose there exist two constants $A \geq 0 \geq B$ with $A \geq|B|$ such that

$$
\begin{array}{ll}
f(t, p, q) \leq A & \text { for } 0 \leq t \leq 1,0 \leq p \leq \ell A, 0 \leq q \leq A / 2, \\
f(t, p, q) \geq B & \text { for } 0 \leq t \leq 1, \ell B \leq p \leq 0,0 \leq q \leq-B / 2 .
\end{array}
$$

If $f(t, p, q)$ is increasing in $p$, then problem (5.1) has a solution $x(t)$ such that

$$
\alpha(t) \leq x(t) \leq \beta(t), \quad \beta^{(3)}(t) \leq x^{(3)}(t) \leq \alpha^{(3)}(t), \quad t \in[0,1],
$$

where

$$
\alpha(t)=B \int_{0}^{1} G(t, s) s d s, \quad \beta(t)=A \int_{0}^{1} G(t, s) s d s .
$$

Proof. From the conditions (5.6) and (5.7), and Lemmas 5.3 and 5.4, problem (5.1) has an upper solution $\beta(t)$ and a lower solution $\alpha(t)$.

Set $\phi=-\alpha^{(3)}$ and $\psi=-\beta^{(3)}$. Then $\phi(t)=B t \leq A t=\psi(t)$. On the other hand, $A \geq 0 \geq B$ implies that $\alpha^{(3)}(t) \geq \beta^{(3)}(t)$. The fact that $G(t, s) \geq 0$ and the boundary condition yield

$$
\alpha(t) \leq \beta(t) \quad \text { for } 0 \leq t \leq 1 .
$$


Now define

$$
\begin{gathered}
D: \operatorname{dom} D=\left\{u \in C^{1}(0,1) \cap C[0,1]: u(0)=0\right\} \rightarrow C[0,1], \\
(D u)(t)=u^{\prime}(t), \quad t \in(0,1),
\end{gathered}
$$

and let $F: C[0,1] \rightarrow C[0,1]$ be the continuous operator defined by

$$
(F u)(t)=f(t, T(\min \{\psi, \max \{u, \phi\}\}), K(\min (\psi, \max \{u, \phi\}))) .
$$

Consider the equation

$$
D u=F u, \quad u \in \operatorname{dom} D .
$$

If $u^{*}$ is a solution of $(5.8)$, then $\phi(t) \leq u^{*}(t) \leq \psi(t)$ for $t \in[0,1]$. Consequently, $u^{*}(t)$ is a solution of (5.5). Furthermore, $u^{*}(t)=\left(T u^{*}\right)(t)$ is a solution of (5.1) satisfying $\alpha(t) \leq u^{*}(t) \leq \beta(t)$. In fact, if $\phi(t)>u^{*}(t)$, then there exists $t_{1} \in[0,1]$ such that $\phi\left(t_{1}\right)>u^{*}\left(t_{1}\right)$. As $u^{*}(0)=-\alpha^{\prime \prime}(0)=\phi(0)=0$, by the continuity of $u^{*}$ and $\phi$, there exists an interval $\left(t_{2}, t_{3}\right)$ such that $\phi\left(t_{2}\right)=u^{*}\left(t_{2}\right)$ and $\phi(t)>u^{*}(t)$ for $t \in\left(t_{2}, t_{3}\right)$. Therefore, for $t \in\left[t_{2}, t_{3}\right]$,

$$
\begin{aligned}
\left(F u^{*}\right)(t) & =f\left(t, T\left(\min \left\{\psi, \max \left\{u^{*}, \phi\right\}\right\}\right), K\left(\min \left\{\psi, \max \left\{u^{*}, \phi\right\}\right\}\right)\right) \\
& =f\left(t, T\left(\min \left\{\psi, \max \left\{u^{*}, \phi\right\}\right\}\right), K(\phi)\right) .
\end{aligned}
$$

Let $k(t)=u^{*}(t)-\phi(t)$. By the monotonicity of $f$ and $T$, one has

$$
\begin{aligned}
\phi^{\prime}(t) & =-\alpha^{(4)}(t) \leq f\left(t, \alpha(t), \alpha^{\prime \prime}(t)\right)=f(t,(T \phi)(t),(K \phi)(t)), \quad t \in[0,1], \\
u^{* \prime}(t) & =\left(F u^{*}\right)(t)=f\left(t, T\left(\min \left\{\psi, \max \left\{u^{*}, \phi\right\}\right\}\right),(K \phi)(t)\right), \quad t \in\left[t_{2}, t_{3}\right] .
\end{aligned}
$$

Then $k^{\prime}(t) \geq 0$ for $t \in\left[t_{2}, t_{3}\right]$. Thus $k\left(t_{2}\right)=0$ implies $k(t) \geq 0$ for $t \in\left[t_{2}, t_{3}\right]$, so $\phi(t) \leq u^{*}(t)$ for $t \in\left[t_{2}, t_{3}\right]$. This contradicts our supposition. Thus, $\phi(t) \leq$ $u^{*}(t)$ for $t \in[0,1]$. The proof of $\psi(t) \geq u^{*}(t)$ for $t \in[0,1]$ is quite similar and is omitted.

Let now $H: C[0,1] \rightarrow[0,1]$, be defined by

$$
(H u)(t)=\int_{0}^{t}(F u)(s) d s, \quad t \in[0,1] .
$$

It is clear that $H$ is a continuous operator, and a fixed point of $H$ is a solution of problem (5.8).

Set $M_{A}=\{\theta \in C[0,1]:\|\theta\| \leq A\}$. It is not difficult to see that $\psi, \phi \in M_{A}$.

We now prove that $H: M_{A} \rightarrow M_{A}$ is a completely continuous operator. From the condition (5.6), one has

$$
|(H u)(t)-(H u)(s)|=\left|\int_{s}^{t}(F u)(r) d r\right| \leq A|t-s| .
$$

That is, $\left\{H\left(M_{A}\right)\right\}$ is equicontinuous and uniformly bounded. From the Arzelà-Ascoli theorem, we know that $H: M_{A} \rightarrow M_{A}$ is a completely continuous operator, and the Schauder fixed-point theorem guarantees that $H$ 
has a fixed point $u^{*} \in M_{A}$. Therefore $x^{*}(t)=\int_{0}^{1} G(t, s) u^{*}(s) d s$ is a solution of problem (5.1) such that

$$
\alpha(t) \leq x^{*}(t) \leq \beta(t), \quad \alpha^{(3)}(t) \leq x^{*(3)}(t) \leq \beta^{(3)}(t), \quad t \in[0,1] .
$$

Now consider problem (5.2). Assume that $f:[0,1] \times \mathbb{R}^{3} \rightarrow \mathbb{R}$ is continuous. Lemmas similar to Lemmas 5.3 and 5.4 can be obtained. It is not difficult to see that the equation of problem (5.2) is equivalent to

$$
\begin{aligned}
& u^{\prime}(t)=f(t,(T u)(t),(K u)(t),-u(t)), \quad 0<t<1, \\
& u(0)=0,
\end{aligned}
$$

where $(T u)(t)=\int_{0}^{1} G(t, s) u(s) d s$ and $(K u)(t)=\int_{t}^{1} u(s) d s$.

Lemma 5.7. If there exists a constant $A \geq 0$ such that

$$
f(t, p, q, r) \leq A \quad \text { for } 0 \leq t \leq 1, p / \ell, 2 q \in[0, A], r \in[-A, 0],
$$

then problem (5.2) has an upper solution.

LEMmA 5.8. If there exist a constant $B \leq 0$ such that

$$
f(t, p, q, r) \geq B \quad \text { for } 0 \leq t \leq 1, p \in[\ell B, 0], 2 q, r \in[0,-B],
$$

then problem (5.2) has a lower solution.

Lemmas 5.7 and 5.8 can be proved similarly to Lemmas 5.3 and 5.4 and so the proof is omitted. An argument similar to the one in Theorem 5.6 provides the following result about problem (5.2).

Theorem 5.9. Suppose there exist constants $A \geq 0 \geq B$ with $A \geq|B|$ such that

$$
\begin{array}{ll}
f(t, p, q, r) \leq A & \text { for } 0 \leq t \leq 1, p / \ell, 2 q \in[0, A], r \in[-A, 0], \\
f(t, p, q, r) \geq B & \text { for } 0 \leq t \leq 1, p \in[\ell B, 0], 2 q, r \in[0,-B] .
\end{array}
$$

If $f(t, p, q, r)$ is increasing in each of $p$ and $q$, then problem (5.2) has a solution $x(t)$ such that

$$
\alpha(t) \leq x(t) \leq \beta(t), \quad \beta^{(3)}(t) \leq x^{(3)}(t) \leq \alpha^{(3)}(t), \quad \forall t \in[0,1],
$$

where

$$
\alpha(t)=B \int_{0}^{1} G(t, s) s d s, \quad \beta(t)=A \int_{0}^{1} G(t, s) s d s, \quad \ell=\frac{1}{8} .
$$

Example 5.10. Consider the problem

$$
\begin{aligned}
& x^{(4)}(t)+\frac{1}{8}\left[t+e^{x(t)}+x^{\prime \prime}(t)\right]=0, \\
& x(0)=x^{\prime}(0)=x^{\prime \prime}(1)=0, \quad x^{(3)}(0)=0 .
\end{aligned}
$$


Let $f(t, p, q)=\frac{1}{8}\left[t+e^{p}+q\right]$. It is easy to check that $A=4$ and $B=0$ satisfy the conditions (5.6)-(5.7), respectively, therefore,

$$
\alpha(t)=0, \quad \beta(t)=4 \int_{0}^{1} G(t, s) s d s
$$

are lower and upper solutions of (5.11). By Theorem 5.6, problem (5.11) has a solution $x^{*}$ such that

$$
0 \leq x^{*}(t) \leq 4 \int_{0}^{1} G(t, s) s d s .
$$

Example 5.11. Consider the problem

$$
\begin{aligned}
& x^{(4)}(t)+\frac{1}{3}\left[t+\ln (1+x(t))+\left(x^{\prime \prime}(t)\right)^{2}-x^{(3)}(t)\right], \\
& x(0)=x^{\prime}(0)=x^{\prime \prime}(1)=0, \quad x^{(3)}(0)=0 .
\end{aligned}
$$

Let $f(t, p, q, r)=\frac{1}{3}\left[t+\ln (1+p)+q^{2}-r\right]$. It is easy to check that $A=1$ and $B=0$ satisfy the conditions (5.9) and (5.10), respectively, therefore,

$$
\alpha(t)=0, \quad \beta(t)=\int_{0}^{1} G(t, s) s d s
$$

are lower and upper solutions of (5.12). By Theorem 5.9, problem (5.12) has a solution $x^{*}$ such that

$$
0 \leq x^{*}(t) \leq \int_{0}^{1} G(t, s) s d s .
$$

Acknowledgments. The author would like to thank the anonymous referees for their valuable suggestions.

\section{References}

[1] R. P. Agarwal and D. O'Regan, Multiplicity results for singular conjugate, focal, and $(n, p)$ problems, J. Differential Equations 170 (2001), 142-156.

[2] D. R. Anderson, Green's functions for third-order generalized right focal problem, J. Math. Anal. Appl. 288 (2003), 1-14.

[3] Z. Bai, Existence of solutions for some third-order boundary-value problems, Electron. J. Differential Equations 2008, no. 25, 6 pp.

[4] K. Deimling, Nonlinear Functional Analysis, Springer, Berlin, 1985.

[5] B. C. Dhage, Local fixed point theory for the sum of two operators in Banach spaces, Fixed Point Theory 4 (2003), 49-60.

[6] B. C. Dhage and J. Henderson, Existence theory for nonlinear functional boundary value problems, Electron. J. Qualit. Theory Differential Equations 2004, no. 1, 15 pp.

[7] J. Dugundji and A. Granas, Fixed Point Theory, Monogr. Math. 61, PWN, Warszawa, 1982. 
[8] W. Feng and J. R. L. Webb, Solvability of m-point boundary value problems with nonlinear growth, J. Math. Anal. Appl. 212 (1997), 467-480.

[9] J. R. Graef, J. Henderson and B. Yang, Positive solutions for a nonlinear higher order boundary-value problem, Electron. J. Differential Equations 2007, no. 45, 10 pp.

[10] J. R. Graef, C. Qian and B. Yang, Positive solutions of a three point boundary value problem for nonlinear differential equations, in: Dynamic Systems and Applications, Vol. 4, Dynamic, Atlanta, GA, 2004, 431-438.

[11] A. Granas, R. B. Guenther and J. W. Lee, Some general existence principles for Carathéodory theory of nonlinear differential equations, J. Math. Pures Appl. 70 (1991), 267-276.

[12] C. P. Gupta, Solvability of a three-point nonlinear boundary value problem for a second order ordinary differential equation, J. Math. Anal. Appl. 168 (1992), 540551.

[13] —, Solvability of an m-point nonlinear boundary value problem for a second order ordinary differential equation, J. Math. Anal. Appl. 189 (1995), 575-584.

[14] J. Henderson, Boundary Value Problems for Functional Differential Equations, World Sci., Singapore, 1985.

[15] J. Henderson and W. N. Hudson, Eigenvalue problem for nonlinear functional differential equations, Comm. Appl. Nonlinear Anal. 3 (1996), 51-58.

[16] J. Henderson and E. R. Kaufmann, Multiple positive solutions for focal boundary problems, Comm. Appl. Anal. 1 (1997), 53-60.

[17] J. Henderson and S. K. Ntouyas, Positive solutions for systems of nth order threepoint nonlocal boundary value problems, Electron. J. Qualit. Theory Differential Equations 2007, no. 18, 12 pp.

[18] N. Kosmatov, Countably many solutions of a fourth order boundary value problem, ibid. 2004, no. 12, 15 pp.

[19] B. Liu, Positive solutions for a nonlinear three-point boundary value problem, Comput. Math. Appl. 44 (2002), 201-211.

[20] R. Ma, Multiple positive solutions for a semipositone fourth order boundary value problem, Hiroshima Math. J. 33 (2003), 217-227.

[21] Y. P. Sun, Nontrivial solution for a three-point boundary-value problem, Electron. J. Differential Equations 2004, no. 111, 10 pp.

[22] B. Yang, Positive solution for a fourth-order boundary value problem, Electron. J. Qualit. Theory Differential Equations 2005, no. 3, 17 pp.

\author{
A. El-Haffaf \\ Département de Mathématiques \\ Faculté des Sciences \\ Université d'Oran \\ B.P. 1524 Es-Senia, Oran, Algeria \\ E-mail: elhaffaf@yahoo.fr \\ elhaffaf1@univ-oran.dz
}

Received March 20, 2008;

received in final form May 22, 2009

(7654) 